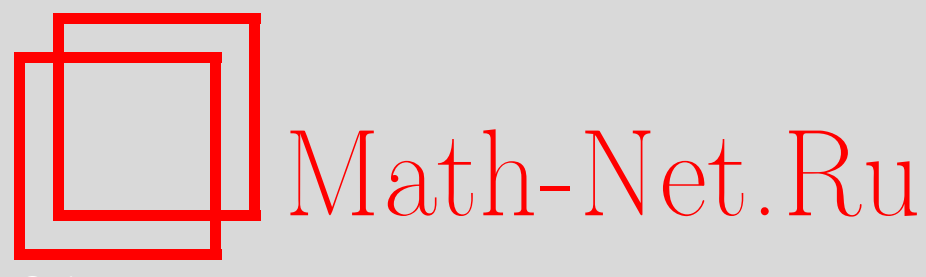

А. В. Латышев, А. А. Юшканов, Продольный электрический ток в столкновительной плазме, генерируемый поперечным электромагнитным полем, ТМФ, 2016, том 187, номер 1, 127-138

DOI: https://doi.org/10.4213/tmf9008

Использование Общероссийского математического портала Math-Net.Ru подразумевает, что вы прочитали и согласны с пользовательским соглашением http: //www . mathnet.ru/rus/agreement

Параметры загрузки:

IP : 34.227 .88 .159

26 апреля 2023 г., 13:48:56

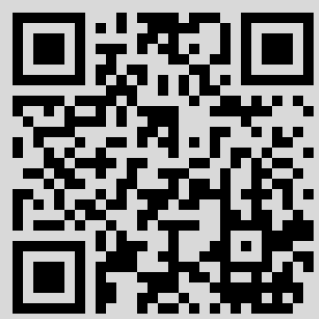




\title{
ПРОДОЛЬНЫЙ ЭЛЕКТРИЧЕСКИЙ ТОК В СТОЛКНОВИТЕЛЬНОЙ ПЛАЗМЕ, ГЕНЕРИРУЕМЫЙ ПОПЕРЕЧНЫМ ЭЛЕКТРОМАГНИТНЫМ ПОЛЕМ
}

\begin{abstract}
Рассматривается столкновительная плазма с произвольной степенью вырождения электронного газа. Плазма находится во внешнем электромагнитном поле. Требуется определить электрический ток, генерируемый в плазме электромагнитным полем. Показано, что электрический ток имеет две ненулевые компоненты. Одна компонента представляет собой поперечный ток, получаемый при линейном анализе. Вторая компонента представляет собой продольный ток, направленный вдоль волнового вектора и ортогональный поперечному току. Исследован случай малых волновых чисел. Когда частота столкновений стремится к нулю, все полученные формулы переходят в формулы, известные для бесстолкновительной плазмы. Проводится графическое исследование безразмерной плотности тока в зависимости от волнового числа, частоты колебаний электромагнитного поля и частоты столкновений электронов с частицами плазмы.
\end{abstract}

Ключевые слова: уравнение Власова, столкновительная плазма, электромагнитное поле, метод последовательных приближений, поперечный и продольный ток.

DOI: $10.4213 / \operatorname{tmf9008}$

\section{1. ВВЕДЕНИЕ}

В настоящей работе выводятся формулы для вычисления электрического тока в столкновительной плазме Ферми-Дирака. Для описания поведения плазмы используется уравнение Власова с интегралом столкновений. В качестве интеграла столкновений используется известный интеграл столкновений Бхатнагара-ГроссаКрука (БГК) [1], [2]. При решении кинетического уравнения Власова, описывающего поведение плазмы, мы учитываем величины, пропорциональные квадрату напряженности внешнего электрического поля. При этом мы используем квадратичные разложения функции распределения, самосогласованного электромагнитного поля и интеграла столкновений.

* Московский государственный областной университет, Москва, Россия. E-mail: avlatyshev@mail.ru,yushkanov@inbox.ru 
При таком нелинейном подходе оказалось, что электрический ток имеет две ненулевые компоненты. Одна компонента электрического тока направлена вдоль напряженности электрического поля и пропорциональна его величине. Эта компонента электрического тока в точности та же самая, что и в линейном анализе. Это поперечный ток. Следовательно, в линейном анализе мы получаем известное выражение поперечного электрического тока. Вторая ненулевая компонента электрического тока пропорциональна квадрату напряженности электрического поля. Она направлена вдоль волнового вектора, перпендикулярно напряженности электрического поля. Этот ток ортогонален первой компоненте тока. Это продольный ток.

Генерирование в плазме продольного тока поперечным электромагнитным полем выявляется при нелинейном анализе взаимодействия электромагнитного поля с плазмой. Нелинейные эффекты в плазме изучаются длительное время [3]-[7].

В работе [4] показано, что вероятности процессов распада могут быть найдены непосредственно из нелинейного тока плазмы. Заметим, что на существование нелинейного тока вдоль волнового вектора указано в работе [5] (см. формулу (2.9) в этой работе).

В экспериментальной работе [8] выяснен вклад нормальной компоненты поля в нелинейный поверхностный ток в сигнале второй гармоники. В работах [9], [10] изучалась генерация нелинейного поверхностного тока при взаимодействии лазерного импульса с металлом. Найдены медленно изменяющиеся во времени нелинейные токи в скин-слое металла, изучено низкочастотное поле, спектральный состав, энергия и форма импульса низкочастотного излучения.

Столкновительная плазма изучалась в работах [11]-[13].

В работах [14]-[16] было исследовано генерирование продольного тока поперечным электромагнитным полем в классической и квантовой бесстолкновительной плазме. В работе [14] рассматривалась плазма Ферми-Дирака, в [15] - максвелловская плазма, а в [16] - вырожденная плазма.

В настоящей работе выведены формулы для вычисления продольного тока, генерируемого поперечным электромагнитным полем, в классической столкновительной плазме при любой температуре, т. е. при любой степени вырожденности электронного газа.

\section{2. РЕШЕНИЕ УРАВНЕНИЯ ВЛАСОВА}

Покажем, что в классической плазме, описываемой уравнением Власова, генерируется продольный ток поперечным электрическим полем, и вычислим его плотность. На существование этого тока указывалось более полувека тому назад [5].

Рассмотрим уравнение Власова, описывающее поведение столкновительной плазмы с интегралом столкновений БГК,

$$
\frac{\partial f}{\partial t}+\mathbf{v} \frac{\partial f}{\partial \mathbf{r}}+e\left(\mathbf{E}+\frac{1}{c}[\mathbf{v}, \mathbf{H}]\right) \frac{\partial f}{\partial \mathbf{p}}=\nu\left(f^{(0)}-f\right) .
$$

Здесь $f$ - функция распределения электронов плазмы, $\mathbf{E}, \mathbf{H}$ - компоненты электромагнитного поля, $c$ - скорость света, $\mathbf{p}=m \mathbf{v}-$ импульс-электронов, $\mathbf{v}-$ скорость электронов, $\nu$ - эффективная частота столкновений электронов с частицами плазмы, $f^{(0)}=f_{\text {eq }}(\mathbf{r}, v)$ - локально-равновесная функция распределения Ферми-Дирака,

$$
f_{\mathrm{eq}}(\mathbf{r}, v)=\left[1+e^{(\mathcal{E}-\mu(\mathbf{r})) / k_{\mathrm{B}} T}\right]^{-1}=\left[1+e^{P^{2}-\alpha(\mathbf{r})}\right]^{-1}=f_{\mathrm{eq}}(\mathbf{r}, P),
$$


$\mathcal{E}=m v^{2} / 2$ - энергия электронов, $\mu(\mathbf{r})$ - химический потенциал электронного газа, $k_{\mathrm{B}}$ - постоянная Больцмана, $T$ - температура плазмы, $\mathbf{P}=\mathbf{P} / p_{T}=\mathbf{v} / v_{T}$ - безразмерный импульс (скорость) электронов, $p_{T}=m v_{T}, v_{T}$ - тепловая скорость электронов, $v_{T}=\sqrt{2 k_{\mathrm{B}} T / m}, \alpha(\mathbf{r})=\mu(\mathbf{r}) / k_{\mathrm{B}} T-$ безразмерный (приведенный) химический потенциал, $k_{\mathrm{B}} T=\mathcal{E}_{T}=m v_{T}^{2} / 2$ - тепловая энергия электронов.

Будем считать, что в плазме имеется электромагнитное поле, представляющее собой бегущую гармоническую волну $\mathbf{E}=\mathbf{E}_{0} e^{i(\mathbf{k} r-\omega t)}, \mathbf{H}=\mathbf{H}_{0} e^{i(\mathbf{k} r-\omega t)}$. Электрическое и магнитное поля связаны между собой векторным потенциалом:

$$
\mathbf{E}=-\frac{1}{c} \frac{\partial \mathbf{A}}{\partial t}, \quad \mathbf{H}=\operatorname{rot} \mathbf{A} .
$$

Для определенности будем считать, что волновой вектор направлен вдоль оси $x$, а электрическое поле направлено вдоль оси $y$, т. е. $\mathbf{k}=k(1,0,0), \mathbf{E}=E_{y}(x, t)(0,1,0)$. Следовательно,

$$
\begin{gathered}
\mathbf{E}=-\frac{1}{c} \frac{\partial \mathbf{A}}{\partial t}=\frac{i \omega}{c} \mathbf{A}, \quad \mathbf{H}=\frac{c k}{\omega} E_{y} \cdot(0,0,1), \quad[\mathbf{v}, \mathbf{H}]=\frac{c k}{\omega} E_{y} \cdot\left(v_{y},-v_{x}, 0\right), \\
e\left(\mathbf{E}+\frac{1}{c}[\mathbf{v}, \mathbf{H}]\right) \frac{\partial f}{\partial \mathbf{p}}=\frac{e}{\omega} E_{y}\left[k v_{y} \frac{\partial f}{\partial p_{x}}+\left(\omega-k v_{x}\right) \frac{\partial f}{\partial p_{y}}\right],
\end{gathered}
$$

а также

$$
[\mathbf{v}, \mathbf{H}] \frac{\partial f_{0}}{\partial \mathbf{p}}=0
$$

так как $\partial f_{0} / \partial \mathbf{p} \sim \mathbf{v}$

Рассмотрим линеаризацию локально-равновесной функции распределения

$$
f_{\mathrm{eq}}(P, x)=f_{0}(P)+g(P) \delta \alpha(x),
$$

где

$$
\begin{gathered}
f_{0}(P)=\left[1+e^{P^{2}-\alpha}\right]^{-1}, \quad \alpha(x)=\alpha+\delta \alpha(x), \quad \alpha=\text { const }, \\
g(P)=\frac{\partial f_{0}(P)}{\partial \alpha}=\frac{e^{P^{2}-\alpha}}{\left(1+e^{P^{2}-\alpha}\right)^{2}} .
\end{gathered}
$$

Уравнение (1) может быть переписано в виде

$$
\frac{\partial f}{\partial t}+v_{x} \frac{\partial f}{\partial x}+\frac{e E_{y}}{\omega}\left[k v_{y} \frac{\partial f}{\partial p_{x}}+\left(\omega-k v_{x}\right) \frac{\partial f}{\partial p_{y}}\right]+\nu f=\nu f_{0}(P)+g(P) \nu \delta \alpha(x) .
$$

Величина $\delta \alpha(x)$ может быть найдена из закона сохранения числа частиц

$$
\int\left(f_{\mathrm{eq}}-f\right) \frac{2 d^{3} p}{(2 \pi \hbar)^{2}}=0
$$

Из этого уравнения получаем

$$
\delta \alpha(x)=\int\left[f-f_{0}(P)\right] d^{3} P\left[\int g(P) d^{3} P\right]^{-1} .
$$

5 Теоретическая и математическая физика, т. 187, № 1, 2016 г. 
Заметим, что

$$
\int g(P) d^{3} P=2 \pi \int_{0}^{\infty} \frac{d P}{1+e^{P^{2}-\alpha}}=\pi \int_{-\infty}^{\infty} \frac{d P}{1+e^{P^{2}-\alpha}}=\pi \hat{f}_{0}(\alpha),
$$

где

$$
\hat{f}_{0}(\alpha)=\int_{-\infty}^{\infty} \frac{d P}{1+e^{P^{2}-\alpha}}=2 \int_{0}^{\infty} \frac{d P}{1+e^{P^{2}-\alpha}}
$$

Следовательно,

$$
\delta \alpha(x)=\frac{1}{\pi \hat{f}_{0}(\alpha)} \int\left[f-f_{0}(P)\right] d^{3} P .
$$

Уравнение (2) может быть преобразовано теперь к интегральному уравнению

$$
\begin{gathered}
\frac{\partial f}{\partial t}+v_{x} \frac{\partial f}{\partial x}+\nu f=\nu f_{0}(P)-\frac{e E_{y}}{\omega}\left[k v_{y} \frac{\partial f}{\partial p_{x}}+\left(\omega-k v_{x}\right) \frac{\partial f}{\partial p_{y}}\right]+ \\
+\nu g(P) \frac{1}{\pi \hat{f}_{0}(\alpha)} \int\left[f-f_{0}(P)\right] d^{3} P
\end{gathered}
$$

Будем искать решение уравнения (3) в виде

$$
f=f_{0}(P)+f_{1}+f_{2},
$$

где

$$
f_{1} \sim E_{y} \sim e^{i(k x-\omega t)}, \quad f_{2} \sim E_{y}^{2} \sim e^{2 i(k x-\omega t)} .
$$

В задаче имеются три параметра размерности длины $l_{1}=v_{T} / \omega, l_{2}=1 / k$ и $l_{3}=$ $v_{T} \tau(\tau=1 / \nu$ - характерное время между двумя последовательными столкновениями электронов). Будем полагать, что на всех длинах $l_{1}, l_{2}$ и $l_{3}$ изменение энергии электрона под действием электрического поля $E$ много меньше тепловой энергии электронов $k_{\mathrm{B}} T$, т. е. будем считать малыми параметры $\alpha_{1}=|e E| v_{T} / \omega k_{\mathrm{B}} T, \alpha_{2}=$ $|e E| / k k_{\mathrm{B}} T$ и $\alpha_{3}=|e E| v_{T} / \nu k_{\mathrm{B}} T$.

Будем действовать методом последовательных приближений, считая, что $\alpha_{j} \ll 1$, $j=1,2,3$. Тогда с помощью соотношения (4) заключаем, что уравнение (3) эквивалентно следующим уравнениям:

$$
\begin{aligned}
\frac{\partial f_{1}}{\partial t}+v_{x} \frac{\partial f_{1}}{\partial x}+\nu f_{1}= & -\frac{e E_{y}}{\omega}\left[k v_{y} \frac{\partial f_{0}}{\partial p_{x}}+\left(\omega-k v_{x}\right) \frac{\partial f_{0}}{\partial p_{y}}\right]+ \\
& +\nu g(P) \frac{1}{\pi \hat{f}_{0}(\alpha)} \int f_{1} d^{3} P
\end{aligned}
$$

и

$$
\begin{aligned}
\frac{\partial f_{2}}{\partial t}+v_{x} \frac{\partial f_{2}}{\partial x}+\nu f_{2}= & -\frac{e E_{y}}{\omega}\left[k v_{y} \frac{\partial f_{1}}{\partial p_{x}}+\left(\omega-k v_{x}\right) \frac{\partial f_{1}}{\partial p_{y}}\right]+ \\
& +\nu g(P) \frac{1}{\pi \hat{f}_{0}(\alpha)} \int f_{2} d^{3} P
\end{aligned}
$$

Из уравнения (5) получаем

$$
\left(\nu-i \omega+i k v_{x}\right) f_{1}=-\frac{e E_{y}}{\omega}\left[k v_{y} \frac{\partial f_{0}}{\partial p_{x}}+\left(\omega-k v_{x}\right) \frac{\partial f_{0}}{\partial p_{y}}\right]+\nu g(P) A_{1},
$$


где

$$
A_{1}=\frac{1}{\pi \hat{f}_{0}(\alpha)} \int f_{1} d^{3} P .
$$

Введем безразмерные параметры

$$
\Omega=\frac{\omega}{k_{T} v_{T}}, \quad y=\frac{\nu}{k_{T} v_{T}}, \quad q=\frac{k}{k_{T}},
$$

где $q$ - безразмерное волновое число, $k_{T}=m v_{T} / \hbar$ - тепловое волновое число, $\Omega-$ безразмерная частота колебаний электромагнитного поля.

В уравнении (7) перейдем к безразмерным параметрам:

$$
i\left(q P_{x}-z\right) f_{1}=-\frac{e E_{y}}{\Omega k_{T} p_{T} v_{T}}\left[q P_{y} \frac{\partial f_{0}}{\partial P_{x}}+\left(\Omega-q P_{x}\right) \frac{\partial f_{0}}{\partial P_{y}}\right]+y g(P) A_{1},
$$

где $z=\Omega+i y=(\omega+i y) / k_{T} v_{T}$. Заметим, что $\partial f_{0} / \partial P_{x} \sim P_{x}, \partial f_{0} / \partial P_{y} \sim P_{y}$. Следовательно,

$$
q P_{y} \frac{\partial f_{0}}{\partial P_{x}}+\left(\Omega-q P_{x}\right) \frac{\partial f_{0}}{\partial P_{y}}=\Omega \frac{\partial f_{0}}{\partial P_{y}} .
$$

Теперь из уравнения (8) находим

$$
f_{1}=\frac{i e E_{y}}{k_{T} p_{T} v_{T}} \cdot \frac{\partial f_{0} / \partial P_{y}}{q P_{x}-z}-i y \cdot \frac{g(P)}{q P_{x}-z} A_{1} .
$$

Подставляя (9) в уравнение (7), получаем равенство

$$
A_{1}\left(1+i y \int \frac{g(P) d^{3} P}{q P_{x}-z}\right)=\frac{i e E_{y}}{k_{T} p_{T} v_{T}} \int \frac{\partial f_{0} / \partial P_{y}}{q P_{x}-z} d^{3} P .
$$

Легко видеть, что интеграл в правой части этого равенства равен нулю. Следовательно, $A_{1}=0$. Значит, согласно (9) функция $f_{1}$ построена и определяется равенством

$$
f_{1}=\frac{i e E_{y}}{k_{T} p_{T} v_{T}} \cdot \frac{\partial f_{0} / \partial P_{y}}{q P_{x}-z} .
$$

Во втором приближении подставим $f_{1}$ согласно (10) в уравнение $(6)$. Получим уравнение

$$
\begin{aligned}
& \left(\nu-2 i \omega+2 i k v_{x}\right) f_{2}=\nu g(P) A_{2}- \\
& \quad-\frac{i e^{2} E_{y}^{2}}{k_{T} p_{T} v_{T} \omega}\left[k v_{y} \frac{\partial}{\partial p_{x}}\left(\frac{\partial f_{0} / \partial P_{y}}{q P_{x}-z}\right)+\left(\omega-k v_{x}\right) \frac{\partial}{\partial p_{y}}\left(\frac{\partial f_{0} / \partial P_{y}}{q P_{x}-z}\right)\right],
\end{aligned}
$$

где

$$
A_{2}=\frac{1}{\pi \hat{f}_{0}(\alpha)} \int f_{2} d^{3} P .
$$

Перейдем в этом уравнении к безразмерным параметрам:

$$
\begin{aligned}
2 i\left(q P_{x}-x-\frac{i y}{2}\right) f_{2}= & -\frac{i e^{2} E_{y}^{2}}{\Omega k_{T}^{2} p_{T}^{2} v_{T}^{2}}\left[q P_{x} \frac{\partial}{\partial P_{x}}\left(\frac{\partial f_{0} / \partial P_{y}}{q P_{x}-z}\right)+\right. \\
& \left.+\left(\Omega-q P_{x}\right) \frac{\partial}{\partial P_{y}}\left(\frac{\partial f_{0} / \partial P_{y}}{q P_{x}-z}\right)\right]+y g(P) A_{2}
\end{aligned}
$$


Вводя обозначение

$$
z^{\prime}=\Omega+\frac{i y}{2}=\frac{\omega}{k_{T} v_{T}}+i \frac{\nu}{2 k_{T} v_{T}}=\frac{\omega+i \nu / 2}{k_{T} v_{T}},
$$

из последнего уравнения находим

$$
\begin{aligned}
f_{2}=- & \frac{e^{2} E_{y}^{2}}{2 k_{T}^{2} p_{T}^{2} v_{T}^{2} \Omega}\left[q P_{y} \frac{\partial}{\partial P_{x}}\left(\frac{\partial f_{0} / \partial P_{y}}{q P_{x}-z}\right)+\frac{\Omega-q P_{x}}{q P_{x}-z} \frac{\partial^{2} f_{0}}{\partial P_{y}^{2}}\right] \frac{1}{q P_{x}-z^{\prime}}- \\
& -\frac{i y}{2} \cdot \frac{g(P)}{q P_{x}-z^{\prime}} A_{2} .
\end{aligned}
$$

Для нахождения $A_{2}$ подставим (12) в (11). Из полученного соотношения находим

$$
A_{2}=-\frac{e^{2} E_{y}^{2}}{2 k_{T}^{2} p_{T}^{2} v_{T}^{2} \Omega} \cdot \frac{J_{1}}{\pi \hat{f}_{0}(\alpha)+(i y / 2) J_{0}},
$$

где

$$
\begin{aligned}
J_{0} & =\int \frac{g(P) d^{3} P}{q P_{x}-z^{\prime}} \\
J_{1} & =\int\left[q P_{y} \frac{\partial}{\partial P_{x}}\left(\frac{\partial f_{0} / \partial P_{y}}{q P_{x}-z}\right)+\frac{\Omega-q P_{x}}{q P_{x}-z} \frac{\partial^{2} f_{0}}{\partial P_{y}^{2}}\right] \frac{d^{3} P}{q P_{x}-z^{\prime}} .
\end{aligned}
$$

Подставляя $A_{2}$ в $(12)$, окончательно находим $f_{2}$ в явном виде:

$$
\begin{aligned}
f_{2}= & -\frac{e^{2} E_{y}^{2}}{2 k_{T}^{2} p_{T}^{2} v_{T}^{2} \Omega}\left[q P_{y} \frac{\partial}{\partial P_{x}}\left(\frac{\partial f_{0} / \partial P_{y}}{q P_{x}-z}\right)+\frac{\Omega-q P_{x}}{q P_{x}-z} \frac{\partial^{2} f_{0}}{\partial P_{y}^{2}}\right] \frac{1}{q P_{x}-z^{\prime}}+ \\
& +\gamma \frac{e^{2} E_{y}^{2}}{2 k_{T}^{2} p_{T}^{2} v_{T}^{2} \Omega} \cdot \frac{g(P)}{q P_{x}-z^{\prime}},
\end{aligned}
$$

где

$$
\gamma=\frac{(i y / 2) J_{1}}{\pi \hat{f}_{0}(\alpha)+(i y / 2) J_{0}}
$$

\section{3. ПЛОТНОСТЬ ЭЛЕКТРИЧЕСКОГО ТОКА}

Найдем плотность электрического тока

$$
\mathbf{j}=e \int \mathbf{v} f \frac{2 d^{3} p}{(2 \pi \hbar)^{3}} .
$$

Из равенств (4)-(6) мы видим, что вектор плотности тока имеет две компоненты: $\mathbf{j}=\left(j_{x}, j_{y}, 0\right)$, где $j_{y}-$ плотность поперечного тока,

$$
j_{y}=e \int v_{y} f \frac{2 d^{3} p}{(2 \pi \hbar)^{3}}=e \int v_{y} f_{1} \frac{2 d^{3} p}{(2 \pi \hbar)^{3}} .
$$

Этот ток направлен вдоль электрического поля, его плотность определяется только первым приближением функции распределения. Второе приближение функции 
распределения не вносит вклада в плотность тока. Плотность поперечного тока определяется равенством

$$
j_{y}=\frac{2 i e^{2} p_{T}^{2}}{(2 \pi \hbar)^{3} k_{T}} E_{y}(x, t) \int \frac{\left(\partial f_{0} / \partial P_{y}\right) P_{y}}{q P_{x}-z} d^{3} P .
$$

Этот ток пропорционален первой степени величины напряженности электрического поля.

Для плотности продольного тока по определению имеем

$$
j_{x}=e \int v_{x} f \frac{2 d^{3} p}{(2 \pi \hbar)^{3}}=e \int v_{x} f_{2} \frac{2 d^{3} p}{(2 \pi \hbar)^{3}}=\frac{2 e v_{T} p_{T}^{3}}{(2 \pi \hbar)^{3}} \int P_{x} f_{2} d^{3} P .
$$

С помощью (6) отсюда получаем

$$
\begin{aligned}
j_{x}=\frac{e^{3} E_{y}^{2} m}{(2 \pi \hbar)^{3} k_{T}^{2} \Omega}\{ & -\int\left[q P_{y} \frac{\partial}{\partial P_{x}}\left(\frac{\partial f_{0} / \partial P_{y}}{q P_{x}-z}\right)+\frac{x-q P_{x}}{q P_{x}-z} \frac{\partial^{2} f_{0}}{\partial P_{y}^{2}}\right] \frac{P_{x}}{q P_{x}-z^{\prime}} d^{3} P+ \\
& \left.+\gamma \int \frac{P_{x} g(P)}{q P_{x}-z^{\prime}} d^{3} P\right\} .
\end{aligned}
$$

В интеграле от второго слагаемого в квадратных скобках в (16) внутренний интеграл по $P_{y}$ равен нулю:

$$
\int_{-\infty}^{\infty} \frac{\partial^{2} f_{0}}{\partial P_{y}^{2}} d P_{y}=\left.\frac{\partial f_{0}}{\partial P_{y}}\right|_{P_{y}=-\infty} ^{P_{y}=+\infty}=0
$$

В первом интеграле в квадратных скобках в (16) внутренний интеграл по $P_{x}$ вычисляется по частям:

$$
\int_{-\infty}^{\infty} \frac{\partial}{\partial P_{x}}\left(\frac{\partial f_{0} / \partial P_{y}}{q P_{x}-z}\right) \frac{P_{x}}{q P_{x}-z^{\prime}} d P_{x}=z^{\prime} \int_{-\infty}^{\infty} \frac{\partial f_{0} / \partial P_{y}}{\left(q P_{x}-z\right)\left(q P_{x}-z^{\prime}\right)^{2}} d P_{x} .
$$

Следовательно, равенство (16) упрощается:

$$
j_{x}=\frac{e^{3} E_{y}^{2} m}{(2 \pi \hbar)^{3} k_{T}^{2} \Omega}\left[-z^{\prime} q \int \frac{P_{y}\left(\partial f_{0} / \partial P_{y}\right)}{\left(q P_{x}-z\right)\left(q P_{x}-z^{\prime}\right)^{2}} d^{3} P+\gamma \int \frac{P_{x} g(P)}{q P_{x}-z^{\prime}} d^{3} P\right] .
$$

Внутренний интеграл по переменной $P_{y}$ вычислим по частям:

$$
\int_{-\infty}^{\infty} P_{y} \frac{\partial f_{0}}{\partial P_{y}} d P_{y}=\left.P_{y} f_{0}\right|_{P_{y}=-\infty} ^{P_{y}=+\infty}-\int_{-\infty}^{\infty} f_{0}(P) d P_{y}=-\int_{-\infty}^{\infty} f_{0}(P) d P_{y} .
$$

Таким образом, получим выражение для продольного тока

$$
j_{x}=\frac{e^{3} E_{y}^{2} m}{(2 \pi \hbar)^{3} k_{T}^{2} \Omega}\left[z^{\prime} q \int \frac{f_{0}(P)}{\left(q P_{x}-z\right)\left(q P_{x}-z^{\prime}\right)^{2}} d^{3} P+\gamma \int \frac{P_{x} g(P)}{q P_{x}-z^{\prime}} d^{3} P\right] .
$$

Внутренний интеграл в плоскости $\left(P_{y}, P_{z}\right)$ вычислим в полярных координатах:

$$
\begin{aligned}
\int \frac{f_{0}(P)}{\left(q P_{x}-z\right)\left(q P_{x}-z^{\prime}\right)^{2}} d^{3} P & =\int_{-\infty}^{\infty} \frac{d P_{x}}{\left(q P_{x}-z\right)\left(q P_{x}-z^{\prime}\right)^{2}} \int_{-\infty}^{\infty} \int_{-\infty}^{\infty} f_{0}(P) d P_{y} d P_{z}= \\
& =\pi \int_{-\infty}^{\infty} \frac{\ln \left(1+e^{\alpha-P_{x}^{2}}\right)}{\left(q P_{x}-z\right)\left(q P_{x}-z^{\prime}\right)^{2}} d P_{x}
\end{aligned}
$$


так как

$$
\int_{-\infty}^{\infty} \int_{-\infty}^{\infty} f_{0}(P) d P_{y} d P_{z}=\pi \ln \left(1+e^{\alpha-P_{x}^{2}}\right)
$$

Кроме того,

$$
\begin{aligned}
\int \frac{P_{x} g(P)}{q P_{x}-z^{\prime}} d^{3} P & =\pi \int_{-\infty}^{\infty} \frac{P_{x} f_{0}\left(P_{x}\right)}{q P_{x}-z^{\prime}} d P_{x}=\pi \int_{-\infty}^{\infty} \frac{\tau}{\left(1+e^{\tau^{2}-\alpha}\right)\left(q \tau-z^{\prime}\right)} d \tau= \\
& =\pi \int_{-\infty}^{\infty} \frac{e^{\alpha-\tau^{2}} \tau}{\left(1+e^{\alpha-\tau^{2}}\right)\left(q \tau-z^{\prime}\right)} d \tau=-\frac{\pi q}{2} \int_{-\infty}^{\infty} \frac{\ln \left(1+e^{\alpha-\tau^{2}}\right)}{\left(q \tau-z^{\prime}\right)^{2}} d \tau
\end{aligned}
$$

Интегралы в равенстве (17) сводятся к одномерным интегралам:

$$
j_{x}=\frac{\pi e^{3} E_{y}^{2} m q}{(2 \pi \hbar)^{3} k_{T}^{2} \Omega}\left[z^{\prime} \int_{-\infty}^{\infty} \frac{\ln \left(1+e^{\alpha-\tau^{2}}\right)}{\left(q P_{x}-z\right)\left(q \tau-z^{\prime}\right)^{2}} d \tau-\frac{\gamma}{2} \int_{-\infty}^{\infty} \frac{\ln \left(1+e^{\alpha-\tau^{2}}\right)}{\left(q \tau-z^{\prime}\right)^{2}} d \tau\right]
$$

Перепишем предыдущее равенство в виде

$$
j_{x}=\frac{\pi e^{3} E_{y}^{2} m q}{(2 \pi \hbar)^{3} k_{T}^{2} \Omega}\left[z^{\prime} J_{12}-\frac{\gamma}{2} J_{02}\right],
$$

где

$$
J_{12}=\int_{-\infty}^{\infty} \frac{\ln \left(1+e^{\alpha-\tau^{2}}\right)}{\left(q P_{x}-z\right)\left(q \tau-z^{\prime}\right)^{2}} d \tau, \quad J_{02}=\int_{-\infty}^{\infty} \frac{\ln \left(1+e^{\alpha-\tau^{2}}\right)}{\left(q \tau-z^{\prime}\right)^{2}} d \tau .
$$

Вернемся к рассмотрению величины $\gamma$. Вычислим интегралы, входящие в (14). Вычислим первый интеграл:

$$
J_{1}=\int\left[q P_{y} \frac{\partial}{\partial P_{x}}\left(\frac{\partial f_{0} / \partial P_{y}}{q P_{x}-z}\right)+\frac{x-q P_{x}}{q P_{x}-z} \frac{\partial^{2} f_{0}}{\partial P_{y}^{2}}\right] \frac{d^{3} P}{q P_{x}-z^{\prime}} .
$$

Как уже указывалось, интеграл от второго слагаемого равен нулю. Второй интеграл, как и ранее, вычислим по частям. В результате получаем

$$
J_{1}=q \int P_{y} \frac{\partial}{\partial P_{x}}\left(\frac{\partial f_{0} / \partial P_{y}}{q P_{x}-z}\right) \frac{d^{3} P}{q P_{x}-z^{\prime}}=q^{2} \int \frac{P_{y}\left[\partial f_{0} / \partial P_{y}\right]}{\left(q P_{x}-z\right)\left(q P_{x}-z^{\prime}\right)^{2}} d^{3} P
$$

Теперь вычислим по частям внутренний интеграл по переменной $P_{y}$ :

$$
J_{1}=-q^{2} \int \frac{f_{0}(P)}{\left(q P_{x}-z\right)\left(q P_{x}-z^{\prime}\right)^{2}} d^{3} P .
$$

Этот интеграл был вычислен ранее. Следовательно,

$$
J_{1}=-\pi q^{2} \int_{-\infty}^{\infty} \frac{\ln \left(1+e^{\alpha-\tau^{2}}\right)}{\left(q P_{x}-z\right)\left(q \tau-z^{\prime}\right)^{2}} d \tau=-\pi q^{2} J_{12}
$$

Вычислим второй интеграл из (14):

$$
J_{0}=\int \frac{g(P)}{q P_{x}-z^{\prime}} d^{3} P=\int_{-\infty}^{\infty} \frac{d P_{x}}{q P_{x}-z^{\prime}} \int_{-\infty}^{\infty} \int_{-\infty}^{\infty} g(P) d P_{y} d P_{z} .
$$


Для внутреннего двойного интеграла получаем

$$
\int_{-\infty}^{\infty} \int_{-\infty}^{\infty} g(P) d P_{y} d P_{z}=\pi \frac{1}{1+e^{P^{2}-\alpha}}=\pi f_{0}(P) .
$$

Следовательно,

$$
J_{0}=\pi \int_{-\infty}^{\infty} \frac{f_{0}(\tau)}{q \tau-z^{\prime}} d \tau
$$

так что

$$
\pi \hat{f}_{0}(\alpha)+\frac{i y}{2} J_{0}=\pi \int_{-\infty}^{\infty} \frac{q \tau-\Omega}{q \tau-z^{\prime}} f_{0}(\tau) d \tau .
$$

Таким образом, константа $\gamma$ найдена:

$$
\gamma=-\frac{i y}{2} q^{2}\left(\frac{J_{12}}{J_{01}}\right)
$$

где

$$
J_{01}=\int_{-\infty}^{\infty} \frac{q \tau-\Omega}{q \tau-z^{\prime}} f_{0}(\tau) d \tau .
$$

Теперь формулу (18) можно представить в виде

$$
j_{x}=\frac{\pi e^{3} E_{y}^{2} m q}{(2 \pi \hbar)^{3} k_{T}^{2} \Omega}\left[\Omega+\frac{i y}{2}+\frac{i y}{4} q^{2} \frac{J_{02}}{J_{01}}\right] J_{12} .
$$

Найдем числовую плотность (концентрацию) частиц плазмы, отвечающую распределению Ферми-Дирака:

$$
N=\int f_{0}(P) \frac{2 d^{3} p}{(2 \pi \hbar)^{3}}=\frac{8 \pi p_{T}^{3}}{(2 \pi \hbar)^{3}} \int_{0}^{\infty} \frac{e^{\alpha-P^{2}} P^{2} d P}{1+e^{\alpha-P^{2}}}=\frac{k_{T}^{3}}{2 \pi^{2}} l_{0}(\alpha)
$$

где

$$
l_{0}(\alpha)=\int_{0}^{\infty} \ln \left(1+e^{\alpha-\tau^{2}}\right) d \tau
$$

В выражении перед интегралом в (19) выделим плазменную (ленгмюровскую) частоту $\omega_{\mathrm{p}}=\sqrt{4 \pi e^{2} N / m}$ и числовую плотность (концентрацию) $N$, последнюю выразим через тепловое волновое число. Получим

$$
j_{x}^{\text {long }}=\left(\frac{e \Omega_{\mathrm{p}}^{2}}{k_{T} p_{T}}\right) \frac{k E_{y}^{2}}{16 \pi l_{0}(\alpha) \Omega}\left[\Omega+\frac{i y}{2}+\frac{i y}{4} q^{2} \frac{J_{02}}{J_{01}}\right] J_{12},
$$

где

$$
\Omega_{\mathrm{p}}=\frac{\omega_{\mathrm{p}}}{k_{T} v_{T}}=\frac{\hbar \omega_{\mathrm{p}}}{m v_{T}^{2}}
$$

- безразмерная плазменная частота.

Равенство (20) перепишем в виде

$$
j_{x}^{\text {long }}=J(\Omega, y, q) \sigma_{1, \operatorname{tr}} k E_{y}^{2},
$$


где $\sigma_{1, \mathrm{tr}}$ - продольно-поперечная проводимость, $J(\Omega, y, q)$ - безразмерная часть тока,

$$
\begin{aligned}
& \sigma_{1, \mathrm{tr}}=\frac{e \Omega_{\mathrm{p}}^{2}}{p_{T} k_{T}}=\frac{e \hbar}{p_{T}^{2}}\left(\frac{\hbar \omega_{\mathrm{p}}}{m v_{T}^{2}}\right)^{2}=\frac{e}{k_{T} p_{T}}\left(\frac{\omega_{\mathrm{p}}}{k_{T} v_{T}}\right)^{2}, \\
& J(\Omega, y, q)=\frac{1}{16 \pi l_{0}(\alpha) \Omega}\left[\Omega+\frac{i y}{2}+\frac{i y}{4} q^{2} \frac{J_{02}}{J_{01}}\right] J_{12} .
\end{aligned}
$$

Если ввести поперечное поле

$$
\mathbf{E}_{\mathrm{tr}}=\mathbf{E}-\frac{\mathbf{k}(\mathbf{E k})}{k^{2}}=\mathbf{E}-\frac{\mathbf{q}(\mathbf{E q})}{q^{2}}, \quad \mathbf{k E}_{\mathrm{tr}}=\frac{\omega}{c}[\mathbf{E}, H],
$$

то равенство (21) можно представить в инвариантной форме

$$
\mathbf{j}^{\text {long }}=J(\Omega, y, q) \sigma_{1, \mathrm{tr}} \mathbf{k E}_{\mathrm{tr}}^{2}=J(\Omega, y, q) \sigma_{\mathrm{l}, \mathrm{tr}} \frac{\omega}{c}[\mathbf{E}, H] .
$$

ЗАмЕчАниЕ 1. Из формулы (19) (или (20)) видно, что при $y=0$ (или $\nu=0$ ), т. е. когда частота столкновений стремится к нулю и плазма становится бесстолкновительной $\left(z \rightarrow \Omega, z^{\prime} \rightarrow \Omega\right)$, эта формула в точности переходит в соответствующую формулу из работы [14] для бесстолкновительной плазмы

$$
j_{x}^{\text {long }}=\sigma_{1, \operatorname{tr}} k E_{y}^{2} \frac{1}{16 \pi l_{0}(\alpha)} \int_{-\infty}^{\infty} \frac{\ln \left(1+e^{\alpha-\tau^{2}}\right)}{(q \tau-\Omega)^{2}(q \tau-\Omega)} d \tau .
$$

Перейдем к рассмотрению случая малых значений волновых чисел. Из выражения (19) при малых значениях волновых чисел получаем

$$
\begin{aligned}
j_{x}^{\text {long }} & =-\sigma_{1, \operatorname{tr}} k E_{y}^{2} \frac{1}{16 \pi l_{0}(\alpha) \Omega z z^{\prime}} \int_{-\infty}^{\infty} \ln \left(1+e^{\alpha-\tau^{2}}\right) d \tau= \\
& =-\frac{\sigma_{1, \operatorname{tr}} k E_{y}^{2}}{8 \pi \Omega z z^{\prime}}=-\frac{e}{8 \pi m \omega}\left(\frac{\omega_{\mathrm{p}}}{\omega}\right)^{2} \frac{k E_{y}^{2}}{(1-i \nu / \omega)(1-i \nu / 2 \omega)} .
\end{aligned}
$$

ЗАМЕЧАНИЕ 2. При $\nu=0$ из формулы (22) в точности вытекает соответствующая формула из работы [14] для продольного тока в случае малых значений волновых чисел в бесстолкновительной плазме.

На рис. 1 показано поведение действительной (рис. 1а) и мнимой (рис. 1б) частей плотности безразмерного продольного тока при $\Omega=1, y=0.01$ в зависимости от безразмерного волнового числа $q$ при различных значениях безразмерного химического потенциала. При малых и больших значениях параметра $q$ кривые для разных значений $\alpha$ сближаются и становятся неразличимыми. Действительная часть имеет сначала минимум, а затем максимум. С ростом безразмерного химического потенциала мнимая часть плотности тока имеет один максимум.

Далее графическое исследование величины плотности продольного тока проведем для случая нулевого химического потенциала: $\alpha=0$ (рис. 2,3$)$. 


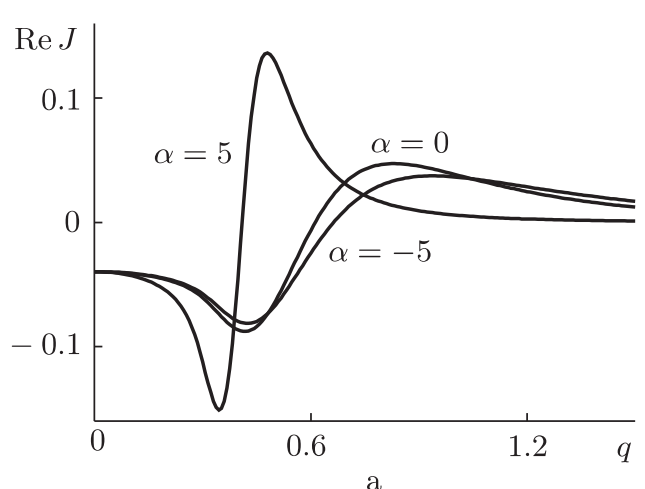

Рис. 1. Действительная (а) и мнимая (б) части плотности безразмерного продольного тока, $\Omega=1, y=0.01$.
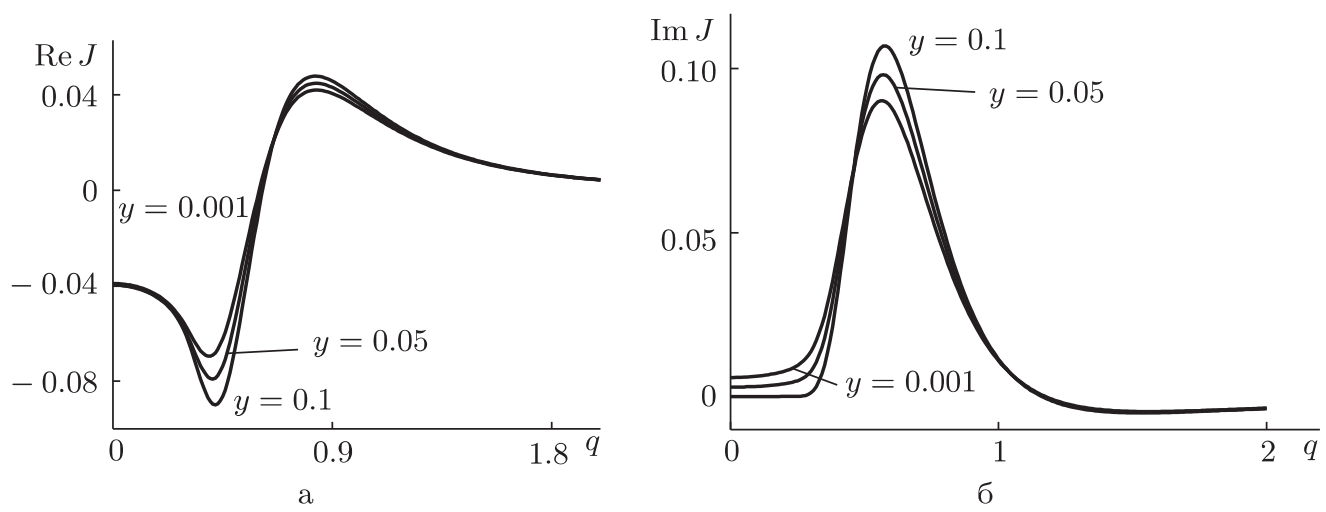

Рис. 2. Действительная (а) и мнимая (б) части плотности безразмерного продольного тока, $\Omega=1, \alpha=0$.

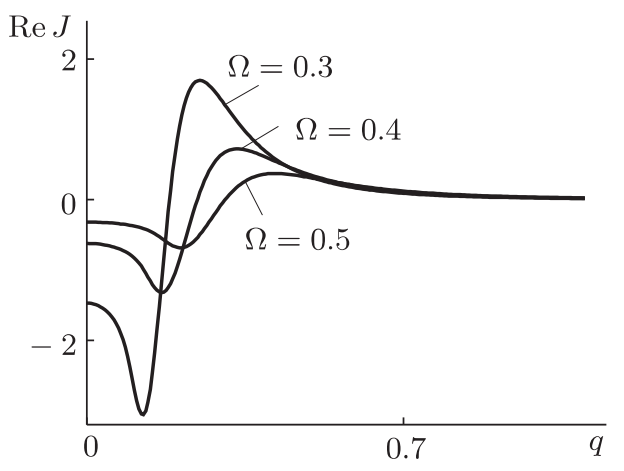

a

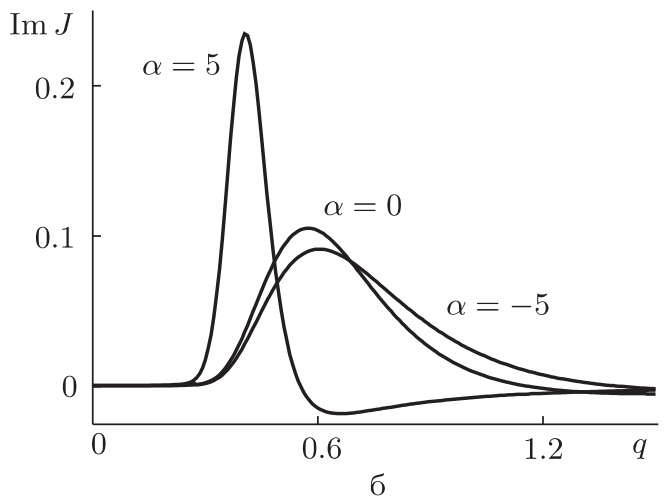


На рис. 2 представлено поведение действительной (рис. 2а) и мнимой (рис. 2б) частей плотности продольного тока в зависимости от безразмерного волнового числа $q$ в случае $\Omega=1$ при различных значениях безразмерной частоты столкновений электронов. При малых и при больших значениях безразмерного волнового числа кривые для разных значений $у$ сближаются и становятся неразличимыми.

На рис. 3 представлено поведение действительной (рис. За) и мнимой (рис. Зб) частей плотности продольного тока в зависимости от безразмерного волнового числа $q$ при различных значениях безразмерной частоты колебаний электромагнитного поля $\Omega$. При возрастании безразмерного волнового числа $q$ кривые для разных значений $\Omega$ сближаются и при $q \geqslant 1$ практически совпадают.

\section{4. ЗАКЛЮЧЕНИЕ}

Рассмотрено влияние нелинейного характера взаимодействия электромагнитного поля с классической столкновительной плазмой. Рассматривается случай произвольной температуры, т. е. произвольной степени вырождения электронного газа.

Оказалось, что наличие нелинейности электромагнитного поля приводит к генерированию электрического тока, ортогонального к направлению поля. Этот продольный ток перпендикулярен к известному поперечному классическому току.

В дальнейшем авторы намерены рассмотреть задачу плазменных колебаний и задачу о скин-эффекте с использованием квадрата векторного потенциала в разложении функции распределения.

\section{Список литературы}

[1] P. L. Bhatnagar, E. P. Gross, M. Krook, Phys. Rev., 94:3 (1954), 511-525.

[2] P. Welander, Ark. Fys., 7 (1954), 507-553.

[3] В. Л. Гинзбург, А. В. Гуревич, УФН, 70:2 (1960), 201-246.

[4] В. Н. Цытович, УФН, 90:11 (1966), 435-489.

[5] Л.М.Коврижных, В.Н. Цытович, ЖЭТФ, 47:4 (1965), 1454-1462.

[6] В. Н. Цытович, Нелинейные эффекты в плазме, Ленанд, М., 2014.

[7] П. К. Шукла, Б. Элиассон, УФН, 180:1 (2010), 55-82.

[8] N. N. Akhmediev, I. V. Mel'nikov, L. J. Robur, Laser Phys., 4:6 (1994), 1194-1197.

[9] С. Г. Бежанов, С. А. Урюпин, Квант. электроника, 43:11 (2013), 1048-1054.

[10] В.Е. Гришков, С. А. Урюпин, "Генерация нелинейного тока вдоль направления распространения короткого лазерного импульса", XLI Международная (Звенигородская) конферениия по физике плазмы и управляемому термоядерному синтезу (10-14 февраля 2014 г.), ЗАО НТЦ "Плазмаиофан", М., 2014, 95.

[11] N. D. Mermin, Phys. Rev. B, 1:5 (1970), 2362-2363.

[12] А. В. Латышев, А. А. Юшканов, ТMФ, 175:1 (2013), 132-143.

[13] А. В. Латышев, А. А. Юшканов, ТМФ, 178:1 (2014), 147-160.

[14] А. В. Латышев, А. А. Юшканов, Физика плазмьи, 41:9 (2015), 778-787.

[15] А. В. Латышев, А. А. Юшканов, Изв. РАН. Сер. Механика жидкости и газа, 50:6 (2015), 126-133.

[16] A. V. Latyshev, A. A. Yushkanov, Generation of longitudinal electric current by transversal electromagnetic field in degenerate plasmas, arXiv: 1503.08700.

Поступила в редакцию 18.07.2015, после доработки 17.10.2015 